\title{
Comunicação
}

\section{Primeiro registro do gênero Toxorhynchites Theobald (Diptera, Culicidae) em Mata Atlântica, Viçosa, Minas Gerais ${ }^{1}$}

\author{
Daniel Simões Albeny ${ }^{2}$, Cassiano Sousa Rosa², Lívia Maria Silva Ataíde², Evaldo Ferreira Vilela ${ }^{3}$
}

\section{RESUMO}

Durante os meses de março e abril de 2007 foi verificada a presença do gênero Toxorhynchites (Diptera, Culicidae) em fragmentos de Mata Atlântica no município de Viçosa, Minas Gerais, Brasil. No experimento foram utilizadas 500 armadilhas de oviposição. Registrando-se, pela primeira vez na região de Viçosa o gênero Toxorhynchites, sendo as espécies T. pusillus (Costa Lima, 1931) e T. theobaldi (Dyar \& Knab, 1906) encontradas pela primeira vez no Estado de Minas Gerais. As espécies relatadas como primeiro registro e sua distribuição são citadas e discutidas.

Palavras-chave: Mosquitos, fragmento de floresta, Toxorhynchites pusillus, Toxorhynchites theobaldi.

\section{ABSTRACT}

\section{First record of genus Toxorhynchites Theobald (Diptera, Culicidae) in Mata Atlântica, Viçosa, Minas Gerais State, Brazil}

The occurrence of the genus Toxorhynchites (Diptera, Culicidae) was evaluated during the months March and April 2007 in fragments of Atlantic Forest in the city of Viçosa, Minas Gerais State, Brazil. A total of 500 oviposition traps were used in the experiment. This is the first record of the genus Toxorhynchites in Viçosa and the first record of the species T. pusillus (Costa Lima, 1931) and T. theobaldi (Dyar \& Knab, 1906) in Minas Gerais State. In this scientific note, the species reported as first record and their distribution are described and discussed.

Key words: Mosquitoes, forest fragment, Toxorhynchites pusillus, Toxorhynchites theobaldi.

\footnotetext{
Recebido para publicação em novembro de 2008 e aprovado em setembro de 2009

${ }^{1}$ Trabalho retirado de dissertação de mestrado defendida em 2008

${ }^{2}$ Graduados em Ciências Biológicas, Mestres. Doutorandos em Entomologia, Departamento de Biologia Animal, Universidade Federal de Viçosa, Av. Peter Henry Rolfs s/n, $36570-$ 000, Viçosa, Minas Gerais, Brasil: E-mail: danielalbeny@gmail.com; csrosa@gmail.com; liviaataide@gmail.com

${ }^{3}$ Engenheiro-agrônomo, Ph. Doctor. Departamento de Biologia Animal, Universidade Federal de Viçosa, Av. Peter Henry Rolfs s/n, 36570-000, Viçosa, Minas Gerais, Brasil. Email: evaldo.vilela@gmail.com
} 


\section{INTRODUÇÃO}

Toxorhynchitinae (Diptera: Culicidae) é uma subfamília com apenas um gênero descrito, Toxorhynchites (Ribeiro, 2005). Atualmente existem 93 espécies catalogadas em todo o mundo (Schreiber, 2007), distribuídas nos subgêneros: Ankylorhynchus Lutz, Lynchiella Lahille, Toxorhynchites Theobald e Afrorhynchus Ribeiro. O gênero apresenta ampla distribuição mundial; a maioria das espécies habita áreas florestadas situadas nas regiões tropicais e poucas são encontradas em regiões temperadas (Schreiber, 2007).

No Brasil, os estudos relacionados à ocorrência e descrição do gênero Toxorhynchites datam do início do século XX. Bourroul (1904) e Goeldi (1905) iniciaram algumas breves descrições sobre a biologia do gênero. Peryassú (1908) descreveu várias espécies de culicídeos no Brasil, incluindo alguns Toxorhynchites. Lutz \& Neiva (1913), fizeram importantes considerações sobre a biologia do gênero e descreveram também algumas espécies. Os estudos posteriores foram conduzidos por Costa Lima (1931) que publicou descrições das espécies existentes até aquela data. Os demais são estudos de revisão taxonômica e chaves para identificação de espécies conduzidos por Lane (1939, 1944, 1951, 1953) e descrição de uma nova espécie por Costa Lima et al. (1962), sendo este uma contribuição para a taxonomia das espécies do gênero Toxorhynchites no Brasil. Nove espécies do gênero foram originalmente descritas no Brasil e quatro em outros países, porém com ocorrência também registrada no Brasil (Tabela 1). Assim, até o momento 13 espécies compõem a fauna do gênero no País.

As espécies do gênero Toxorhynchites têm despertado o interesse de vários pesquisadores em todo o mundo (Collins \& Blackwell 2000), o que se deve ao fato de que as larvas são predadoras e podem ser utilizadas no controle biológico de outras larvas que acarretam problemas de saúde pública (Steffan \& Evenhuis, 1981; Jones \& Schreiber, 1994). Elas habitam micro-habitats aquáticos como ocos de árvore, entrenós de bambu, folhas centrais e axilares de bromélias, frutos de sapucaia etc. Nesses ambientes alimentam-se de formas imaturas de macroinvertebrados, preferencialmente larvas de outros culicídeos (Steffan \& Evenhuis, 1981; Jones \& Schreiber, 1994). Os adultos não apresentam hábitos hematófagos (Steffan \& Evenhuis, 1981; Collins \& Blackwell, 2000; Focks, 2007), logo são incapazes de transmitir diretamente agentes patogênicos ao homem (Schreiber, 2007). Consequentemente, várias espécies do gênero estão sendo testadas e avaliadas em programas de controle biológico em todo o mundo (Bonnet \& Hu, 1951; Sempala, 1983; Miyagi et al., 1992).

\section{MATERIAL E MÉTODOS}

O município de Viçosa situa-se na região da Zona da Mata no Estado de Minas Gerais, o clima é tropical de altitude do tipo Cwb, segundo a classificação de Köppen. O estudo foi realizado durante os meses de março e abril de 2007. Larvas do gênero Toxorhynchites em ambientes florestais geralmente são encontradas em ocos de árvores que possam acumular água (Steffan \& Evenhuis, 1981). Devido à grande dificuldade de se encontrar em criadouros naturais (ex.: ocos de árvores) em mata muito densa, foram colocadas 500 armadilhas de oviposição, sendo 100 em cada um dos cinco diferentes fragmentos de Mata Atlântica situados no município. As armadilhas foram dispostas no nível do solo ao longo de quatro transectos de 50 metros cada. Logo, 25 armadilhas foram posicionadas em cada transecto a uma distância de $2 \mathrm{~m}$ uma da outra. Cada transecto foi separado por uma distância de $5 \mathrm{~m}$. Os fragmentos de mata foram denominados: Mata da Biologia (área 1) (2045’ S, $\left.42^{\circ} 51^{\prime} \mathrm{O}\right)$, Mata do seu Nico (área 2)

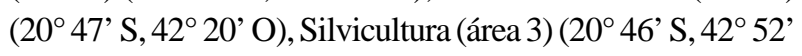
O), Mata do Chaves (área 4) ( $20^{\circ} 43^{\prime} \mathrm{S}, 42^{\circ} 51^{\prime} \mathrm{O}$ ) e Mata do Aeroporto (área 5) ( $20^{\circ} 44^{\prime} \mathrm{S}, 42^{\circ} 50^{\prime} \mathrm{O}$ ). As armadilhas foram confeccionadas a partir de recipientes plásticos de poliestireno $(7 \times 8 \mathrm{~cm})$ que possuíam em seu centro um orifício de $3 \mathrm{~cm}$ de diâmetro. Em cada armadilha foram adicionados $100 \mathrm{ml}$ de água desclorada. As armadilhas foram visitadas a cada 15 dias, para verificação da presença de larvas de Toxorhynchites. Durante todo o experimento foram realizadas quatro coletas em cada área.

Ao final do experimento, foram coletadas, nas respectivas armadilhas, 45 larvas de $4^{\circ}$ ínstar do gênero Toxorhynchites. Devido às limitações taxonômicas existentes na identificação de imaturos do referido gênero, optouse pela identificação das formas adultas. As larvas de Toxorhynchites foram então conduzidas ao laboratório e acondicionadas individualmente em recipientes plásticos de $250 \mathrm{ml}$ onde permaneceram até a emergência dos adultos. As demais espécies animais capturadas nas armadilhas não foram identificadas, mas serviram como alimento para as larvas de Toxorhynchites em suas primeiras horas no laboratório. Posteriormente, larvas de Toxorhynchites foram alimentadas com larvas do mosquito Aedes aegypti (Linnaeus, 1762) (Diptera: Culicidae). As larvas de A. aegypti foram obtidas de ovos provenientes da criação massal do Laboratório de Ecologia Química de Vetores (Departamento de Parasitologia da UFMG). Das 45 larvas coletadas, 23 chegaram à fase adulta, sendo 12 machos e 11 fêmeas. Os adultos foram identificados segundo a chave taxonômica de Lane (1953). As espécies foram enviadas para confirmação pelo Dr. Mário Antônio Navarro da Silva (Universidade Federal do Paraná). O material coletado e identificado foi depositado no Museu Regional de Entomologia da Universidade Federal de Viçosa (UFV). 


\section{RESULTADOS E DISCUSSÃO}

Foram identificadas duas espécies a partir do material coletado: a) Toxorhynchites (Lynchiella) theobaldi (Dyar \& Knab, 1906) sendo sete exemplares, três machos e quatro fêmeas, coletados nas áreas 1, 2, 3, 4 e 5; e b) Toxorhynchites (Lynchiella) pusillus (Costa Lima, 1931), sendo oito exemplares, cinco machos e três fêmeas, coletados nas áreas 2, 4 e 5. Os demais exemplares, quatro machos e quatro fêmeas, não puderam ser identificados com precisão. Entretanto, seguindo-se a chave taxonômica de Lane (1953), esses exemplares foram por exclusão, considerados como T. theobaldi ou T. pusillus.

A espécie T. pusillus foi descrita por Costa Lima (1931) com o holótipos coletados no Alto da Boa Vista, Tijuca, Rio de Janeiro, Brasil. É uma espécie originalmente descrita no Brasil (Tabela 1), não havendo registro de ocorrência dela em outros países e tampouco sinônimos para essa espécie (Costa Lima, 1931; Lane, 1944, 1953; Belkin et al.,
1971). Os holótipos estão depositados no Instituto Osvaldo Cruz (IOC). Não havia registros de ocorrência da espécie para o estado de Minas Gerais até o presente momento.

A espécie T. theobaldi foi descrita por Dyar \& Knab (1906), com base em dois espécimes, um macho e uma fêmea coletados por Theobald na cidade de Bogotá, Colômbia, depositados no British Museum (BM) (Belkin, 1968). A espécie tem ampla distribuição nas Américas do Sul e Central, sendo encontrada no Brasil, Argentina, Belize, Bolívia, Colômbia, Costa Rica, Equador, El Salvador, Guatemala, Guiana, México, Nicarágua, Panamá, Suriname, Trinidad e Tobago, Uruguai e Venezuela (Lane, 1953; Pecor et al., 2002). Provavelmente devido a sua ampla distribuição e a coleta de espécimes em diferentes áreas geográficas, a espécie apresenta várias outras sinonímias (Tabela 2). No entanto, não havia na literatura nenhum registro de sua ocorrência no Estado de Minas Gerais.

Tabela 1: Espécies do gênero Toxorhynchites originalmente descritas no Brasil e espécies do gênero Toxorhynchites descritas em outros países, com ocorrencia registrada no Brasil

\begin{tabular}{llll}
\hline Espécies & \multicolumn{1}{c}{ Autores } & P. de descrição & \multicolumn{1}{c}{ Referências } \\
\hline T. bambusicola & (Lutz \& Neiva) & Brasil & Lutz \& Neiva (1913) \\
T. catharinensis & (Da Costa Lima, Guitton \& Ferreira) & Brasil & Costa Lima et al. (1962) \\
T. mariae & (Bourroul) & Brasil & Bourroul (1904) \\
T. purpureus & (Theobald) & Brasil & Theobald (1901) \\
T. pussilus & (Da Costa Lima) & Brasil & Costa Lima (1931) \\
T. rizzoi & (Deus Palma \& Bello Galvao) & Brasil & Silva et al. (1996) \\
T. solstitialis & (Lutz) & Brasil & Belkin (1968); Belkin et al. (1971) \\
T. trichopygus & (Wiedemann) & Brasil & Belkin (1968); Belkin et al. (1971) \\
T. violaceus & (Wiedemann) & Brasil & Costa Lima (1931) \\
T. guadeloupensis & (Dyar \& Knab) & Guadalupe & Lane (1953) \\
T. haemorrhoidalis & (Fabricius) & Guiana Francesa & Lane (1953) \\
T. separatus & (Lynch) & Argentina & Belkin (1968) \\
T. theobaldi & (Dyar \& Knab) & Bolivia & Dyar \& Knab (1906) \\
\hline
\end{tabular}

Tabela 2: Sinonímias, autor, país, local de coleta e museus onde estão depositadas as espécies sinônimas de Tx. theobaldi

\begin{tabular}{llllll}
\hline Sinonímia & Autor & País & Local da Coleta & Depositores & Referências \\
\hline $\begin{array}{l}\text { T. ferox } \\
\text { T. } \text { wiedemanni }\end{array}$ & (Wiedmann) & Brasil & Salvador, BA & SNG e NMW ${ }^{1}$ & Belkin (1968) \\
$\begin{array}{l}\text { T. } \text { ambiguus } \\
\text { (1971) }\end{array}$ & (Dyar \& Knab) & $*$ & $* *$ & LU e NMW $^{1}$ & Belkin (1968) \\
T. moctezuma & Brasil & Salvador, BA & USNM $^{1}$ & Belkin (1968); Belkin et al \\
T. trinidadensis & (Dyar \& Knab) & Costa Rica & Puntarenas & USNM $^{1}$ & Lane (1953) \\
T. hypoptes & (Knab) & Trinidade & $*$ & USNM $^{1}$ & Costa Lima (1931) \\
T. fluminensis & (Peryassú) & Nicarágua & Bluefields & USNM $^{1}$ & Lane (1951) \\
T. posticatus & (Lutz \& Neiva) & Brasil & Rio de Janeiro & IOC $^{1}$ & Belkin (1968); Lane (1953) \\
T. moengoensis & (Wepster \& Bonne) & Suriname & Petrópolis, RJ & IOC $^{1}$ & Belkin et al (1971) \\
\hline
\end{tabular}

${ }^{1}$ SNG (Natur-Museum und Forschungs-Institut Senckenberg, Senckenberg- Anlage 25, 6 Frankfurt 1); NMW (Naturhistorisches Museum, Burgring 7, Wien 1); LU (Location unknown); USNM (U. S. National Museum, Washington, D. C.); IOC (Instituto Osvaldo Cruz); ITH (Instituut voor Tropische Hygiene, Mauritskade 57, Amsterdam-O).

* País desconhecido.

** Local de coleta desconhecido. 


\section{CONCLUSÕES}

O primeiro registro da ocorrência dos mosquitos Toxorhynchites pusillus e T. theobaldi na região de Viçosa, no Estado de Minas Gerais, fornece oportunidades para futuros estudos, como o estabelecimento de uma criação em massa na Universidade Federal de Viçosa para que ensaios biológicos, principalmente testes de predação de larvas dessas espécies sobre larvas de Aedes aegypti, possam ser realizados.

\section{REFERÊNCIAS}

Belkin JN (1968) The type specimens of new world mosquitoes in European museums. Contribuituion Ammerican Entomological Institute, 3:1-69.

Belkin JN, Schick R \& Heinemann SJ (1971) Mosquitoes originally described from Brazil. Contribution American Entomological Institute, 7:1-63.

Bonnet DD \& Hu SMK (1951) The introduction of Toxorhynchites brevipalpis into the territory of Hawaii. Proceedings Hawaiian Entomological Society, 14:237-242.

Bourroul C (1904) Mosquitos do Brasil. Tese de Doutorado, Salvador, Bahia, 200p.

Collins LE \& Blackwell A (2000) The biology of Toxorhynchites mosquitoes and their potential as biocontrol agents. Biocontrol News and Information, 21:105-116.

Costa Lima A (1931) Sobre as espécies de Megarhinus do Brasil (Díptera: Culicidae). Memórias do Instituto Osvaldo Cruz, 25:307-315.

Costa Lima AM, Guitton N \& Ferreira O (1962) Comentários relativos as espécies da tribo Toxorhynchitini (Megarhinini) com a descrição de uma espécie nova de Lynchiella (Diptera: Culicidae). Memórias do Instituto Osvaldo Cruz, 2:225-255.

Dyar HG \& Knab F (1906) The species of mosquitoes in the genus Megarhinus. Smithsonian Miscellaneous Collection, 48:241-258.

Focks DA (2007) Toxorhynchites as biocontrol agents. Journal of the American Mosquito Control Association, 23:118-127.

Goeldi AE (1905) Os mosquitos do Pará. Memorias do Museu Goeldi, 6:124-127.

Jones CJ \& Schreiber ET (1994) The carnivores, Toxorhynchites. Wing Beats, 5:4-7.
Lane J (1939) Catálogo dos Mosquitos Neotrópicos. Tese de Doutorado. Instituto de Higiene de São Paulo, São Paulo, 211p.

Lane J (1944) A tribo Megarhinini no Brasil Meridional. Revista de Entomologia, 15:172-190.

Lane J (1951) Synonymy of neotropical culicidae (Diptera). Proceedings Entomological Society of Washington, 53:333-336.

Lane J (1953) A tribo Megarhinini no Brasil Meridional, Serie Monográfica. Universidade de São Paulo, São Paulo, 548p.

Lutz A \& Neiva A (1913) Contribuição para o estudo das Megarininas com descrições de duas espécies novas. Memórias do Instituto Osvaldo Cruz, 5:1-15.

Miyagi I, Toma T \& Mogi M (1992) Biological control of container-breeding mosquitoes, Aedes albopictus and Culex quinquefasciatus, in a japanese island by release of Toxorhynchites splendens adults. Medical and Veterinary Entomology, 6:290-300.

Pecor JE, Harbach RE, Peyton EL, Donald TM, Robert R, Rejmankova E, Magnun S \& Palanco J (2002) Mosquito studies in Belize, Central America: Records, taxonomic notes, and a checklist of species. Journal of the American Mosquito Control Association, 18:241-276.

Peryassú AG (1908) Os Culicídeos do Brasil. Rio de Janeiro, Typographia Leuzinger, 407p.

Ribeiro H (2005) Systematics and identification of Afrotropical Toxorhynchitinae (Diptera: Culicidae). Journal of Medical Entomology, 6:953-958.

Schreiber ET (2007) Toxorhynchites. American Mosquito Control Association, 23:129-132.

Sempala SDK (1983) Interactions between immature Aedes africanus (Theobald) and larvae of two predatory species of Toxorhynchites (Diptera, Culicidae) in Zika Forest, Uganda. Bulletin of Entomological Research, 73:19-24.

Silva MV, Oliveira RL, Almeida MD, Vasconselos AS \& Costa J (1996) The type specimens of mosquitoes (Diptera, Culicidae) deposited in the Entomological Collection of the Institute Oswaldo Cruz, Rio de Janeiro, Brazil. Memorias do Instuto Osvaldo Cruz, 91:471-478.

Steffan WA \& Evenhuis NL (1981) Biology of Toxorhynchites. Annual Review of Entomology, 26:159-181.

Theobald FV (1901) A monograph of the Culicidae or mosquitoes. British Museum (Natural History), London, 434p. 